\title{
A Novel Approach to Surface Defect Detection
}

\author{
Yihui $\mathrm{Da}^{1}$, Guirong Dong ${ }^{2}$, Bin Wang ${ }^{1}$, Dianzi Liu ${ }^{3 *}$, Zhenghua Qian $^{1 *}$ \\ 1 State Key Laboratory of Mechanics and Control of Mechanical Structures, College of Aerospace Engineering, \\ Nanjing University of Aeronautics and Astronautics, Nanjing, China \\ 2 Faculty of High Vocational Education, Xi'an University of Technology, Xi'an, China \\ 3 School of Mathematics, University of East Anglia, Norwich, UK, NR4 7TJ
}

${ }^{*}$ Corresponding authors (dianzi.liu@uea.ac.uk; qianzh@nuaa.edu.cn)

\begin{abstract}
Defects or flaws in highly loaded structures have a significant impact on the structural integrity. Early inspection of faults can reduce the likelihood of occurrence of potential disasters and limit the damaging effects of destructions. According to our previous work, a novel approach called as Quantitative Detection of Fourier Transform (QDFT) using guided ultrasonic waves is developed in this paper for efficiently detecting defects in pipeline structures. Details of this fast method consist of three steps: First, an in-house finite element code has been developed to calculate reflection coefficients of guided waves travelling in the pipe. Then, based on boundary integral equations and Fourier transform of space-wavenumber domain, theoretical formulations of the quantitative detection are derived as a function of wavenumber using Born approximation. This lays a solid foundation for QDFT method, in which a reference model in a problem with a known defect is utilized to effectively evaluate the unknown defects. Finally, the location and shape of the unknown defect are reconstructed using signal processing for noise removal. Several examples are presented to demonstrate the correctness and efficiency of the proposed methodology. It is concluded that the general two-dimensional surface defects can be detected with high level of accuracy by this fast approach.
\end{abstract}

\section{Keywords}

Quantitative detection of Fourier Transform, Reconstruction of defects, Hybrid FEM, Boundary integral equation, Reference model 


\section{Introduction}

Quantitative detection of defects in elastic solid is always a hot research topic, and how to precisely determine the defect location and shape is the focus. Early in the ultrasonic inspection, the diffraction tomography (Devaney, 1984; Blackledge, 1987) of the scalar fields was applied, based on full-space Green's function and the equation of scatter fields, to inspect defects in solid, where locations of the source and receiver were different. Subsequently, Blackledge et al. (1987) used quantitative diffraction tomography of elastic waves to reconstruct two-dimensional defects. The tomography methods reconstruction was also applied to inspect concrete structures with and without reinforcement (Chang et al., 2018). In order to reduce the costs of samplings and experiments, and enhance the image resolution, many researchers (Laroque et al., 2008; Batenburg and Sijbers, 2011; Sheppard and Shan, 2010) proposed different algorithms to improve diffraction tomography. In the inverse problems, other approaches were given, which were suitable for different models, such as eddy current methods (Dobson and Santosa, 1998), time reversal (Minnaar and Zhou, 2004) and modal power flow (Wang et al., 2009).

Recently, ultrasonic guided waves have been also used to detect defects, because they are more suitable and effective for large structures inspection. For plate-like structures, Leonard et al. (2002) applied tomographic reconstruction for nondestructive evaluation (NDE) of aerospace structures. Huthwaite and Simonetti (2013) and Huthwaite (2014) compared the ray tomography and diffraction tomography techniques, and provided a mechanism to determine the thickness from a velocity reconstruction. Jing et al. (2017) showed accurate reconstruction of guided wave tomography using full waveform inversion and Hosoya et al. (2018) proposed a non-contact, non-destructive method to generate Lamb waves by laser-induced plasma.

It is challenging work to consider defect inspection in pipeline structures due to the difficulty of extracting singe mode from multiple modes of waves. How to excite useful guided waves in pipelines by experiments is the first step during the process of detecting pipes. Ditri and Rose (1992) applied surface tractions and the normal mode expansion technique to excite guided waves in hollow cylinders. The flexible PVDF pipe comb transducers were designed (Hay and Rose, 2002), which can enhance axial displacements and 
reduce radial displacements. Liu et al. (2006) utilized thickness shear mode piezoelectric elements to excite $\mathrm{T}(0,1)$ mode. In the meanwhile, numerical calculations were performed to simulate scattering fields by many researchers. A combination of finite element formulation and wave function expansion were employed to investigate the scattering of axisymmetric guided waves (Rattanawangcharoen et al., 1997). Duan and Kirby (2015) applied a weighted residual formulation to deliver an efficient hybrid numerical formulation. Mountassir et al. (2018) suggested a Structural Health Monitoring (SHM) method for damage detection and localization in pipeline by calculating a sparse estimation of the current signal. To further apply guided waves for defect inspection in pipes, several detecting methods have been proposed. By frequency bandpass filters and wavelet analysis, Siqueira et al. (2004) processed ultrasonic signals with a low signal/noise ratio acquired with a single transducer in an pulse-echo configuration. Stoyko et al. (2014) found dispersive guided waves can be used to detect a notch in pipes due to the difference of the cutoff frequencies between undamaged pipes and damaged pipes. Employing a circumferentially distributed phased array, guided wave focusing techniques (Mu et al., 2007) were used to detect axial and circumferential location, which improved penetration power and circumferential resolution. An ant colony classification model was proposed to detect structural defects in piles by evaluating displacement-time plots to improve the reliability of pile monitoring (Psychas et al., 2016).

In this paper, we adopt reflection coefficients in full wavenumber domain to reconstruct the pipes' defects using boundary integral equation of ultrasonic waves. To improve surface defect detection, an in-house hybrid finite element (FE) code is developed to efficiently calculate reflection coefficients in scattering fields. Following this, a new approach, called as Quantitative Detection of Fourier Transform (QDFT), is proposed for reconstruction of defects with high level of accuracy and efficiency. The procedure of QDFT for defect detection consists of three steps: first, Fourier transform is applied to convert the shape function $\eta_{0}(x)$ of a known defect into a wavenumber domain function $H_{0}(k)$. Then, reflection wave coefficients $C_{0}(k)$ are calculated by hybrid FEM. Based on boundary integral equation, the term $B(k)$, which only depends on wavenumbers for a given thickness of the structure, is approximately obtained from the reference model. Finally, employing inverse Fourier transform, the real defect $\eta(x)$ is reconstructed. It is noted that the general 
two-dimensional surface defects can be detected with high level of accuracy by the developed QDFT.

\section{Brief review of boundary integral equations for scattering problems}

According to reciprocal theorem (Schmerr, 1998), the integral equation of total fields in Cartesian coordinate system is written as:

$$
\begin{aligned}
& \int_{S}\left[u_{i}^{\text {total }}(\boldsymbol{x}) T_{i j}^{\alpha}(\boldsymbol{x}-\boldsymbol{X})-U_{i}^{\alpha}(\boldsymbol{x}-\boldsymbol{X}) \sigma_{i j}^{\text {total }}(\boldsymbol{x})\right] \boldsymbol{n}_{j} d S(\boldsymbol{x})=u_{\alpha}^{\mathrm{sca}}(\boldsymbol{X}) \\
& i, j, \alpha=1,2 \quad \boldsymbol{X} \notin V
\end{aligned}
$$

where the field point and source point are defined by the coordinates $\left(x_{1}, x_{2}\right)$ and $\left(X_{1}, X_{2}\right)$, respectively. The superscripts 'total' and 'sca' mean total fields and scatter fields, $T_{i j}^{\alpha}(\boldsymbol{x}-\boldsymbol{X})$ and $U_{i}^{\alpha}(\boldsymbol{x}-\boldsymbol{X})$ represent full-space Green's functions of stresses and displacements, $\alpha$ is the direction of a unit load in Green's function, $u_{i}^{\text {total }}(\boldsymbol{x})$ and $\sigma_{i j}^{\text {total }}(\boldsymbol{x})$ are displacements and stresses, $\boldsymbol{n}_{j}$ denotes the normal vector of the defect boundary $S(\boldsymbol{x})$, and ' $V$ ' depicts the defect area. Considering the traction-free boundary condition and Green's function $\tilde{T}_{i j}^{\alpha}(\boldsymbol{x}-\boldsymbol{X})$ of the structure, displacements in scatter fields can be written as:

$\int_{S}\left[u_{i}^{\text {total }}(\boldsymbol{x}) \tilde{T}_{i j}^{\alpha}(\boldsymbol{x}-\boldsymbol{X})\right] \boldsymbol{n}_{j} d S(\boldsymbol{x})=u_{\alpha}^{\text {sca }}(\boldsymbol{X}) \quad \boldsymbol{X} \notin V$

In light of Gauss's divergence theorem, Eq. (2) can be further defined as

$\int_{-\infty}^{+\infty} e^{-2 i \xi x_{2}} e^{i \xi X_{2}} \int_{h-\Delta h_{n}\left(x_{2}\right)-\eta\left(x_{2}\right)}^{h}\left[A_{i}^{\mathrm{inc}}\left(x_{1}\right) \tilde{P}_{i j}^{\alpha}\left(x_{1}-X_{1}\right)\right]_{, j} d x_{1} d x_{2}=u_{\alpha}^{\mathrm{sca}}(\boldsymbol{X})$

where $\xi$ denotes wavenumber, the subscript ',$j$ ' denotes $\frac{\partial}{\partial x_{j}}, \tilde{T}_{i j}^{\alpha}(\boldsymbol{x}-\boldsymbol{X})=\tilde{P}_{i j}^{\alpha}\left(x_{1}-\right.$ $\left.X_{1}\right) e^{-i \xi\left(x_{2}-X_{2}\right)}, h$ and $\eta\left(x_{2}\right)$ signify the structure thickness and defect depth, respectively. According to Born approximation, the total fields can be approximately replaced by the incident fields. Therefore, $A_{i}^{\text {total }}\left(x_{1}\right) \approx A_{i}^{\text {inc }}\left(x_{1}\right), u_{i}^{\text {total }}(\boldsymbol{x}) \approx A_{i}^{\text {inc }}\left(x_{1}\right) e^{-i \xi x_{2}}$, and $\eta\left(x_{2}\right) \rightarrow$ 0 if the defect is small. The incident and reflected waves with the same mode of the guided wave are written as $u_{\alpha}^{\mathrm{inc}}(\boldsymbol{X})=A_{\alpha}^{\mathrm{inc}}\left(X_{1}\right) e^{-i \xi X_{2}}$ and $u_{\alpha}^{\mathrm{ref}}(\boldsymbol{X})=A_{\alpha}^{\mathrm{ref}}\left(X_{1}\right) e^{i \xi X_{2}}$.

When $\boldsymbol{X}$ is far from the defect domain and lies in the reflection region, the displacements in the scattered fields are represented by the ones in the reflected fields:

$u_{\alpha}^{\mathrm{sca}}(\boldsymbol{X})=u_{\alpha}^{\mathrm{ref}}(\boldsymbol{X})=A_{\alpha}^{\mathrm{ref}}\left(X_{1}\right) e^{i \xi X_{2}=C^{\mathrm{ref}}}(\xi) A_{\alpha}^{\mathrm{inc}}\left(X_{1}\right) e^{i \xi X_{2}}$ 
where the reflection coefficients $C^{\mathrm{ref}}(\xi)=\frac{A_{\alpha}^{\mathrm{ref}}\left(X_{1}\right)}{A_{\alpha}^{\mathrm{inc}}\left(X_{1}\right)}$.

Since the integrand $\left[A_{i}^{\text {total }}\left(x_{1}\right) \tilde{P}_{i j}^{\alpha}\left(x_{1}-X_{1}\right)\right]_{, j}$ has no singularity, its original function must exist as follows

$\left.\widetilde{W}\left(x_{1}, \xi\right)\right|_{\mathrm{h}-\eta\left(x_{2}\right)} ^{\mathrm{h}}=\int_{\mathrm{h}-\eta\left(x_{2}\right)}^{\mathrm{h}}\left[A_{i}^{\mathrm{total}}\left(x_{1}\right) \tilde{P}_{i j}^{\alpha}\left(x_{1}-X_{1}\right)\right]_{, j} d x_{1}$

Considering Eqs. (4) and (5), Eq. (3) can be rewritten as:

$$
\begin{aligned}
\int_{-\infty}^{+\infty} e^{-2 i \xi x_{2}} e^{i \xi x_{2}} \eta\left(x_{2}\right) \lim _{\eta\left(x_{2}\right) \rightarrow 0} \frac{\widetilde{W}(\mathrm{~h}, \xi)-\widetilde{W}\left(\mathrm{~h}-\eta\left(x_{2}\right), \xi\right)}{\mathrm{h}-\left(\mathrm{h}-\eta\left(x_{2}\right)\right)} d x_{2} \\
\left.\approx \int_{-\infty}^{+\infty} e^{-2 i \xi x_{2}} e^{i \xi x_{2}} \eta\left(x_{2}\right) \frac{d \widetilde{W}\left(x_{1}, \xi\right)}{d x_{1}}\right|_{x_{1}=\mathrm{h}} d x_{2} \approx C^{\mathrm{ref}}(\xi) A_{\alpha}^{\mathrm{inc}}\left(X_{1}\right) e^{i \xi x_{2}}
\end{aligned}
$$

Substituting $R(k)$ with $\left.\frac{d \widetilde{w}\left(x_{1}, \xi\right)}{d x_{1}}\right|_{x_{1}=\mathrm{h}}$ in Eq. (6), one has

$\int_{-\infty}^{+\infty} \eta\left(x_{2}\right) e^{-2 i \xi x_{2}} d x_{2} \approx \frac{C^{\mathrm{ref}}(k) A_{\alpha}^{\mathrm{inc}}\left(X_{1}\right)}{R(k)}$

Finally, the defect depth is obtained by the inverse Fourier transform

$\eta\left(x_{2}\right) \approx \frac{1}{2 \pi} \int_{-\infty}^{+\infty} C^{\mathrm{ref}}(k) B(k) e^{i k x_{2}} d k$

where $B(k)=\frac{A_{\alpha}^{\mathrm{inc}}\left(X_{1}\right)}{R(k)}$ and $k=2 \xi$. It is also noted that the derivation of Eq. (8) has been done in the framework of the zero-order asymptotic modeling, which was introduced in details by Argatov and Butcher (2011).

\section{Determination of reflection coefficients using in-house hybrid FEM}

In Section 2, the relationship between the defect function $\eta\left(x_{2}\right)$ and reflection coefficients $C^{\text {ref }}(k)$ has been described well by Eq. (8). This section mainly introduces how to precisely and efficiently solve the $C^{\mathrm{ref}}(k)$ by a hybrid finite element method (HFEM), which was developed by Stoyko et al. (2014) to detect a notch in a pipe. The early research work by Zhuang and Shah (1999) applied a similar approach to solve the fundamental solution of laminated anisotropic circular cylinder, and the further work was given by Marzani (2008). To improve the computational efficiency of simulations for reflection coefficients, an in-house HFEM based approach is developed in this paper and its formations are given with details in the following Section. 


\subsection{Formulation of HFEM}

In Fig. $1, r_{\text {in }}$ and $r_{\text {out }}$ signify inner and outer diameters, $\mathrm{h}$ represents the wall thickness. The width and depth of the axisymmetric defect are depicted as $l_{a y}$ and $d_{a y}$, respectively. The pipe is truncated by two cross sections $S_{1}$ and $S_{2}$ along the $\mathrm{z}$ axis and the position of the two ends are presented as $z_{S_{1}}$ and $z_{S_{2}}$. The boundaries of the defect are denoted as $z_{L}$ and $z_{R}$. The incident wave propagating along the negative direction of $z$ axis is generated. Based on conventional FEM, the equation of motion for an isotropic elastic medium is written as:

$\delta\left([\mathbf{q}]^{\mathrm{H}}\right) \mathbf{S q}=\delta\left([\mathbf{q}]^{\mathrm{H}}\right) \mathbf{P}$

where

$\mathbf{S}=\mathbf{K}-\omega^{2} \mathbf{M}$

$\mathbf{K}$ and $\mathbf{M}$ are the global stiffness and mass matrices, $\omega$ represents the circular frequency, and $\mathbf{q}$ and $\mathbf{P}$ are nodal displacements and force vectors, respectively. The superscript ' $\mathrm{H}$ ' means conjugate transpose and ' $\delta$ ' denotes the first variation. As shown in Fig. 1, the pipeline is modelled by 20 nodal hexahedron isoparametric elements. All nodes are divided into two categories: one group includes the boundary nodes located at the cross sections $S_{1}$ and $S_{2}$, which are marked by subscript 'B'; the other group consists of the interior nodes, represented by subscript 'I'.

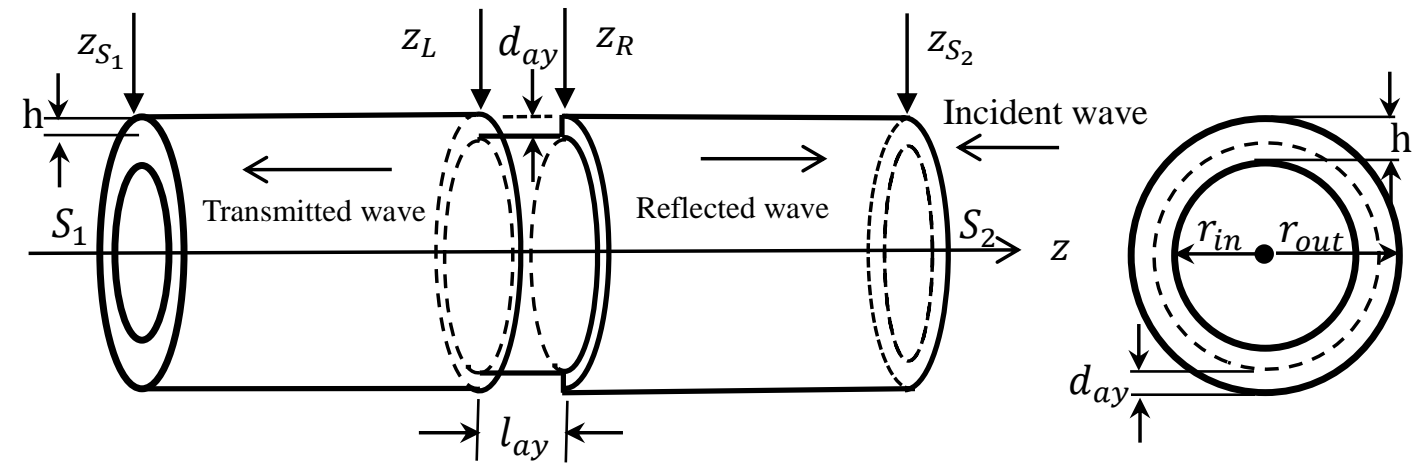

Fig. 1 A pipe with an axisymmetric defect

Rewriting Eq. (9) in matrix form, one can have

$\delta\left(\left[\begin{array}{c}\mathbf{q}_{\mathrm{I}} \\ \mathbf{q}_{\mathrm{B}}\end{array}\right]^{\mathrm{H}}\right)\left[\begin{array}{cc}\mathbf{S}_{\mathrm{II}} & \mathbf{S}_{\mathrm{IB}} \\ \mathbf{S}_{\mathrm{BI}} & \mathbf{S}_{\mathrm{BB}}\end{array}\right]\left[\begin{array}{c}\mathbf{q}_{\mathrm{I}} \\ \mathbf{q}_{\mathrm{B}}\end{array}\right]=\delta\left(\left[\begin{array}{c}\mathbf{q}_{\mathrm{I}} \\ \mathbf{q}_{\mathrm{B}}\end{array}\right]^{\mathrm{H}}\right)\left[\begin{array}{c}\mathbf{P}_{\mathrm{I}} \\ \mathbf{P}_{\mathrm{B}}\end{array}\right]$

where $\mathbf{P}_{\mathrm{I}}=\mathbf{0}$. The nodal displacement vector $\mathbf{q}_{\mathrm{B}}=\left\{\begin{array}{l}\mathbf{q}_{S_{1}} \\ \mathbf{q}_{S_{2}}\end{array}\right\}$ and force vector $\mathbf{P}_{\mathrm{B}}=\left\{\begin{array}{l}\mathbf{P}_{S_{1}} \\ \mathbf{P}_{S_{2}}\end{array}\right\}$ at 
cross sections $S_{1}$ and $S_{2}$ can be decomposed into incidence, reflection and transmission components as below:

$\mathbf{q}_{S_{1}}=\mathbf{q}_{S_{1}}^{\mathrm{inc}}+\mathbf{q}_{S_{1}}^{\mathrm{tra}}$

$\mathbf{q}_{S_{2}}=\mathbf{q}_{S_{2}}^{\mathrm{inc}}+\mathbf{q}_{S_{2}}^{\mathrm{ref}}$

and

$\mathbf{P}_{S_{1}}=\mathbf{P}_{S_{1}}^{\mathrm{inc}}+\mathbf{P}_{S_{1}}^{\mathrm{tra}}$

$\mathbf{P}_{S_{2}}=\mathbf{P}_{S_{2}}^{\text {inc }}+\mathbf{P}_{S_{2}}^{\text {ref }}$

where the superscripts 'inc', 'tra' and 'ref' indicate the incident, transmitted and reflected waves, respectively.

The displacement in pipelines can be presented by Fourier series in the $\theta$ direction:

$\mathbf{U}=\sum_{n=-\infty}^{+\infty} e^{i n \theta} \overline{\mathbf{U}}_{n}(z)$

where $\overline{\mathbf{U}}_{n}(z)$ depicts the displacement for each circumferential mode $n$ including the propagating and non-propagating waves. It is noted that in this paper only $n=0$ is considered as the mode of the incident wave, i.e., circumferential symmetrical modes. According to different displacement forms, circumferential symmetrical modes are divided into the extensional mode $\mathrm{L}(0, m)$ and the torsional mode $\mathrm{T}(0, m)$. In the cylindrical coordinate system $(r, \theta, z), \mathrm{L}(0, m)$ includes displacements both in $r$ and $z$ directions, and $\mathrm{T}(0, m)$ is only related to displacements in $\theta$ direction. Therefore, the nodal displacements of the incident waves $\mathbf{q}^{\text {inc }}$ are written as

$\mathbf{q}^{\text {inc }}=-\frac{i}{2 \pi r_{0}} \frac{k_{01}\left[\boldsymbol{\Phi}_{01 u}^{L}\right]^{\mathrm{H}} \mathbf{F}_{0}}{B_{01}} \boldsymbol{\phi}_{01 u}^{R} e^{-i k_{01}\left(z-z_{0}\right)}=\boldsymbol{\Phi}_{01} e^{-i k_{01}\left(z-z_{0}\right)}$

and the nodal forces are derived from the constitutive (stress-strain) relationship of the elastic material

$\mathbf{P}^{\text {inc }}=\left\{\mathbf{D}\left[\left(\boldsymbol{B}_{1}-i k_{01} \boldsymbol{B}_{3}\right) \boldsymbol{\Phi}_{01}\right]\right\} \boldsymbol{n} e^{-i k_{01}\left(z-z_{0}\right)}=\mathbf{t}^{\mathrm{inc}} e^{-i k_{01}\left(z-z_{0}\right)}$

where $k_{01}$ indicates the wavenumber of the $\mathrm{T}(0,1)$ mode, $\mathbf{D}$ is a $(6 \times 6)$ matrix of cylindrically isotropic elastic moduli, $\boldsymbol{n}$ means the normal vector of the cross sections, $z_{0}$ is the location of source $\mathbf{F}_{0}$, and the other symbols can be found in the work (Zhuang and Shah, 1999).

According to Cook's work for a pipe with axisymmetric defects (Cook, 1981), the 
circumferential wavenumbers of the transmitted and reflected waves are required to be identical to those of the incident waves, i.e., the transmitted and reflected waves are only associated with $0^{\text {th }}$ circumferential mode for the $\mathrm{T}(0,1)$ incident mode. On the cross sections $S_{1}$ and $S_{2}$, the transmitted and reflected wave fields can be written in the summation forms

$$
\begin{aligned}
& \mathbf{q}_{S_{1}}^{\operatorname{tra}}=\sum_{m=1}^{3(2 \mathrm{M}+1)}-\frac{i A_{0 m}^{\mathrm{tra}}}{2 \pi r_{0}} \frac{k_{0 m}\left[\boldsymbol{\Phi}_{0 m u}^{L}\right]^{\mathrm{H}} \mathbf{F}_{0}}{B_{0 m}} \boldsymbol{\phi}_{0 m u}^{R} e^{-i k_{0 m} z}=\sum_{m=1}^{3(2 \mathrm{M}+1)} A_{0 m}^{\mathrm{tra}} \boldsymbol{\Phi}_{0 m}^{\mathrm{tra}} e^{-i k_{0 m} z} \\
& \mathbf{P}_{S_{1}}^{\mathrm{tra}}=\sum_{m=1}^{3(2 \mathrm{M}+1)} \mathbf{D}\left[A_{0 m}^{\mathrm{tra}}\left(\boldsymbol{B}_{1}-i k_{0 m} \boldsymbol{B}_{3}\right) \boldsymbol{\Phi}_{0 m}^{\mathrm{tra}} e^{-i k_{0 m} z}\right] \boldsymbol{n}=\sum_{m=1}^{3(2 \mathrm{M}+1)} A_{0 m}^{\mathrm{tra}} \mathbf{t}_{0 m}^{\mathrm{tra}} e^{-i k_{0 m} z}
\end{aligned}
$$

and

$$
\begin{aligned}
& \mathbf{q}_{S_{2}}^{\mathrm{ref}}=\sum_{m=1}^{3(2 \mathrm{M}+1)}-\frac{i A_{0 m}^{\mathrm{ref}}}{2 \pi r_{0}} \frac{k_{0 m}\left[\boldsymbol{\phi}_{0 m u}^{L}\right]^{\mathrm{H}} \mathbf{F}_{0}}{B_{0 m}} \boldsymbol{\phi}_{0 m u}^{R} e^{i k_{0 m} z}=\sum_{m=1}^{3(2 \mathrm{M}+1)} A_{0 m}^{\mathrm{ref}} \boldsymbol{\Phi}_{0 m}^{\mathrm{ref}} e^{i k_{0 m} z} \\
& \mathbf{P}_{S_{2}}^{\mathrm{ref}}=\sum_{m=1}^{3(2 \mathrm{M}+1)} \mathbf{D}\left[A_{0 m}^{\mathrm{ref}}\left(\boldsymbol{B}_{1}+i k_{0 m} \boldsymbol{B}_{3}\right) \boldsymbol{\Phi}_{0 m}^{\mathrm{ref}} e^{i k_{0 m} z}\right] \boldsymbol{n}=\sum_{m=1}^{3(2 \mathrm{M}+1)} A_{0 m}^{\mathrm{ref}} \mathbf{t}_{0 m}^{\mathrm{ref}} e^{i k_{0 m} z}
\end{aligned}
$$

where $\mathrm{M}$ is the number of the radial discrete elements, $A_{0 m}^{\text {ref }}$ and $A_{0 m}^{\text {tra }}$ are the unknown reflected and transmitted coefficients.

In Eqs.(19)-(22), the wavenumber is real for propagating waves and complex for non-propagating waves. When $k_{0 m}$ is purely imaginary or complex, the attenuation characteristics of non-propagating waves can not be accurately expressed in form of $e^{i k_{0 m} z}$ due to the potential large value of the exponential term. However, based on the fact that the defect in the pipelines can be treated as a scattering source generating the transmitted and reflected waves, the minimum attenuation distance can be defined by the defect location and cross sections. Therefore, Eqs.(19)-(22) are modified as follows:

$$
\begin{gathered}
\mathbf{q}_{S_{1}}^{\text {tra }}=\sum_{m=1}^{3(2 \mathrm{M}+1)}\left(A_{0 m}^{\mathrm{tra}} e^{-i k_{0 m} z_{L}}\right) \boldsymbol{\Phi}_{0 m}^{\mathrm{tra}} e^{-i k_{0 m}\left(z_{S_{1}}-z_{L}\right)} \approx \sum_{m=1}^{\widetilde{\mathrm{M}}} \tilde{A}_{0 m}^{\mathrm{tra}} \boldsymbol{\Phi}_{0 m}^{\mathrm{tra}} e^{-i k_{0 m}\left(z_{S_{1}}-z_{L}\right)} \\
\mathbf{P}_{S_{1}}^{\mathrm{tra}}=\sum_{m=1}^{3(2 \mathrm{M}+1)} \mathbf{D}\left[\tilde{A}_{0 m}^{\operatorname{tra}}\left(\boldsymbol{B}_{1}-i k_{0 m} \boldsymbol{B}_{3}\right) \boldsymbol{\Phi}_{0 m}^{\mathrm{tra}} e^{-i k_{0 m}\left(z_{S_{1}}-z_{L}\right)}\right] \\
\approx \sum_{m=1}^{\widetilde{\mathrm{M}}} \tilde{A}_{0 m}^{\operatorname{tra}} \mathbf{t}_{0 m}^{\mathrm{tra}} e^{-i k_{0 m}\left(z_{S_{1}}-z_{L}\right)}
\end{gathered}
$$

and 
$\mathbf{q}_{S_{2}}^{\mathrm{ref}}=\sum_{m=1}^{3(2 \mathrm{M}+1)}\left(A_{0 m}^{\mathrm{ref}} e^{i k_{0 m} z_{R}}\right) \boldsymbol{\Phi}_{0 m}^{\mathrm{ref}} e^{i k_{0 m}\left(z_{S_{2}}-z_{R}\right)} \approx \sum_{m=1}^{\widetilde{\mathrm{M}}} \tilde{A}_{0 m}^{\mathrm{ref}} \boldsymbol{\Phi}_{0 m}^{\mathrm{ref}} e^{i k_{0 m}\left(z_{S_{2}}-z_{R}\right)}$

$\mathbf{P}_{S_{2}}^{\mathrm{ref}}=\sum_{m=1}^{3(2 \mathrm{M}+1)} \mathbf{D}\left[\tilde{A}_{0 m}^{\mathrm{ref}}\left(\boldsymbol{B}_{1}+i k_{0 m} \boldsymbol{B}_{3}\right) \boldsymbol{\Phi}_{0 m}^{\mathrm{ref}} e^{i k_{0 m}\left(z_{S_{2}}-z_{R}\right)}\right] \approx \sum_{m=1}^{\widetilde{\mathrm{M}}} \tilde{A}_{0 m}^{\mathrm{ref}} \mathbf{t}_{0 m}^{\mathrm{ref}} e^{i k_{0 m}\left(z_{S_{2}}-z_{R}\right)}$

It is noted that $\widetilde{\mathrm{M}} \leq 3(2 \mathrm{M}+1)$ is observed by ignoring the wavenumber $k_{0 m}$ with a large imaginary part. Also, $\tilde{A}_{0 m}^{\text {tra }}$ and $\tilde{A}_{0 m}^{\text {ref }}$ are unknown coefficients in the above equations.

Substituting Eqs.(17), (18), and (23)-(26) into Eqs. (12)-(15), the following equations can be derived

$\mathbf{q}_{S_{1}} \approx \boldsymbol{\Phi}_{0 m} e^{-i k_{0 m}\left(z_{S_{1}}-z_{0}\right)}+\sum_{m=1}^{\widetilde{\mathrm{M}}} \tilde{A}_{0 m}^{\mathrm{tra}} \boldsymbol{\Phi}_{0 m}^{\mathrm{tra}} e^{-i k_{0 m}\left(z_{S_{1}}-z_{L}\right)}=\widetilde{\boldsymbol{\Phi}}_{0 m}^{1}+\sum_{m=1}^{\widetilde{\mathrm{M}}} \tilde{A}_{0 m}^{\mathrm{tra}} \widetilde{\boldsymbol{\Phi}}_{0 m}^{\mathrm{tra}}$

$\mathbf{q}_{S_{2}} \approx \boldsymbol{\Phi}_{0 m} e^{-i k_{0 m}\left(z_{S_{2}}-z_{0}\right)}+\sum_{m=1}^{\widetilde{\mathrm{M}}} \tilde{A}_{0 m}^{\mathrm{ref}} \boldsymbol{\Phi}_{0 m}^{\mathrm{ref}} e^{i k_{0 m}\left(z_{S_{2}}-z_{R}\right)}=\widetilde{\boldsymbol{\Phi}}_{0 m}^{2}+\sum_{m=1}^{\widetilde{\mathrm{M}}} \tilde{A}_{0 m}^{\mathrm{ref}} \widetilde{\boldsymbol{\Phi}}_{0 m}^{\mathrm{ref}}$

and

$\mathbf{P}_{S_{1}} \approx \mathbf{t}^{\mathrm{inc}} e^{-i k_{0 m}\left(z_{S_{1}}-z_{0}\right)}+\sum_{m=1}^{\widetilde{\mathrm{M}}} \tilde{A}_{0 m}^{\mathrm{tra}} \mathbf{t}_{S_{1}}^{\mathrm{tra}} e^{-i k_{0 m}\left(z_{S_{1}}-z_{L}\right)}=\mathbf{t}_{0 m}^{1}+\sum_{m=1}^{\widetilde{\mathrm{M}}} \tilde{A}_{0 m}^{\mathrm{tra}} \tilde{\mathbf{t}}_{0 m}^{\mathrm{tra}}$

$\mathbf{P}_{S_{2}} \approx \mathbf{t}^{\mathrm{inc}} e^{-i k_{0 m}\left(z_{S_{2}}-z_{0}\right)}+\sum_{m=1}^{\widetilde{\mathrm{M}}} \tilde{A}_{0 m}^{\mathrm{ref}} \mathbf{t}_{S_{2}}^{\mathrm{ref}} e^{i k_{0 m}\left(z_{S_{2}}-z_{R}\right)}=\mathbf{t}_{0 m}^{2}+\sum_{m=1}^{\widetilde{\mathrm{M}}} \tilde{A}_{0 m}^{\mathrm{ref}} \tilde{\mathbf{t}}_{0 m}^{\mathrm{ref}}$

Finally, the equation of motion is reformulated as follows:

$[\mathbf{G}]\left[\begin{array}{l}\mathbf{q}_{\mathrm{I}} \\ \widetilde{\mathbf{A}}\end{array}\right]=[\mathbf{T}]$

where $\mathbf{G}=\left\{\left[\begin{array}{cc}\mathbf{I} & \mathbf{0} \\ \mathbf{0} & {[\widetilde{\boldsymbol{\Phi}}]^{\mathrm{H}}}\end{array}\right]\left[\begin{array}{cc}\mathbf{S}_{\mathrm{II}} & \mathbf{S}_{\mathrm{IB}} \\ \mathbf{S}_{\mathrm{BI}} & \mathbf{S}_{\mathrm{BB}}\end{array}\right]\left[\begin{array}{cc}\mathbf{I} & \mathbf{0} \\ \mathbf{0} & \widetilde{\boldsymbol{\Phi}}\end{array}\right]-\left[\begin{array}{cc}\mathbf{I} & \mathbf{0} \\ \mathbf{0} & {[\widetilde{\boldsymbol{\Phi}}]^{\mathrm{H}}}\end{array}\right]\left[\begin{array}{cc}\mathbf{0} & \mathbf{0} \\ \mathbf{0} & \tilde{\mathbf{t}}\end{array}\right]\right\}, \mathbf{T}=\left[\begin{array}{cc}\mathbf{I} & \mathbf{0} \\ \mathbf{0} & {[\widetilde{\boldsymbol{\Phi}}]^{\mathrm{H}}}\end{array}\right]\left\{\left[\begin{array}{c}0 \\ \tilde{\mathbf{t}}^{1}\end{array}\right]-\right.$

$\left.\left[\begin{array}{cc}\mathbf{S}_{\mathrm{II}} & \mathbf{S}_{\mathrm{IB}} \\ \mathbf{S}_{\mathrm{BI}} & \mathbf{S}_{\mathrm{BB}}\end{array}\right]\left[\begin{array}{c}0 \\ \widetilde{\boldsymbol{\Phi}}^{1}\end{array}\right]\right\}, \widetilde{\mathbf{A}}=\left[\begin{array}{c}\tilde{A}_{01}^{\text {tra }} \\ \vdots \\ \tilde{A}_{0 \widetilde{\mathrm{M}}}^{\mathrm{tra}} \\ \tilde{A}_{01}^{\mathrm{ref}} \\ \vdots \\ \tilde{A}_{0 \widetilde{\mathrm{M}}}^{\mathrm{raf}}\end{array}\right], \quad \widetilde{\boldsymbol{\Phi}}=\left[\begin{array}{llllll}\widetilde{\boldsymbol{\Phi}}_{01}^{\mathrm{tra}} & \ldots & \widetilde{\boldsymbol{\Phi}}_{0 \widetilde{\mathrm{M}}}^{\mathrm{tra}} & \widetilde{\boldsymbol{\Phi}}_{01}^{\mathrm{ref}} & \ldots & \widetilde{\boldsymbol{\Phi}}_{0 \widetilde{\mathrm{M}}}^{\mathrm{ref}}\end{array}\right], \quad \widetilde{\boldsymbol{\Phi}}^{1}=\left[\begin{array}{c}\widetilde{\boldsymbol{\Phi}}_{0 m}^{1} \\ \widetilde{\boldsymbol{\Phi}}_{0 m}^{2}\end{array}\right]$,

$\tilde{\mathbf{t}}^{1}=\left[\begin{array}{l}\mathbf{t}_{0 m}^{1} \\ \mathbf{t}_{0 m}^{2}\end{array}\right], \quad \tilde{\mathbf{t}}=\left[\begin{array}{llllll}\tilde{\mathbf{t}}_{01}^{\mathrm{tra}} & \cdots & \tilde{\mathbf{t}}_{0 \overline{\mathrm{M}}}^{\mathrm{tra}} & \tilde{\mathbf{t}}_{01}^{\mathrm{ref}} & \cdots & \tilde{\mathbf{t}}_{0 \overline{\mathrm{M}}}^{\mathrm{ref}}\end{array}\right], \quad \mathbf{I}$ is an identity matrix, $\mathbf{q}_{\mathrm{I}}$ is the displacement vector of the interior nodes and $\widetilde{\mathbf{A}}$ is the modified coefficients for scattered fields. 
Taking into account the shape function $\mathbf{N}$ in FEM, displacements and forces of any particle in pipelines can be determined with respect to different modes

$u_{0 m}^{\text {tra }}=\tilde{A}_{0 m}^{\text {tra }} \mathbf{N} \widetilde{\boldsymbol{\Phi}}_{0 m}^{\text {tra }} ; f_{0 m}^{\text {tra }}=\tilde{A}_{0 m}^{\text {tra }} \mathbf{N} \tilde{\mathbf{t}}_{0 m}^{\text {tra }}$

and

$u_{0 m}^{\mathrm{ref}}=\tilde{A}_{0 m}^{\mathrm{ref}} \mathbf{N} \widetilde{\boldsymbol{\Phi}}_{0 m}^{\mathrm{ref}} ; f_{0 m}^{\mathrm{ref}}=\tilde{A}_{0 m}^{\mathrm{ref}} \mathbf{N} \tilde{\mathbf{t}}_{0 m}^{\mathrm{ref}}$

When $\mathrm{T}(0,1)$ is used for the incident waves, transmitted and reflected coefficients are obtained

$R_{01}^{\text {tra }}=\frac{u_{01}^{\text {tra }}}{u_{01}^{\text {inc }}}$

and

$C_{01}^{\mathrm{ref}}=\frac{u_{01}^{\mathrm{ref}}}{u_{01}^{\mathrm{inc}}} e^{-2 i k_{01} z}$

In Fig. 1, energy carried by the guided waves is stored in the closed region bounded by $S_{1}$, $\mathrm{S}_{2}$, and inner and outer cylindrical surfaces being traction-free. And the time-average values of energy flux in $S_{1}$ and $S_{2}$ can be written as

$\mathrm{E}^{\mathrm{tra}}=\sum_{t=1}^{\mathrm{NP}} \iint_{S_{1}} \operatorname{conj}\left(f_{0 t}^{\mathrm{tra}}+f_{0 m}^{\mathrm{inc}} \delta_{m t}\right)\left(\dot{u}_{0 t}^{\mathrm{tra}}+\dot{u}_{0 m}^{\mathrm{inc}} \delta_{m t}\right) \mathrm{d} S$

and

$E^{\mathrm{ref}}=\sum_{t=1}^{\mathrm{NP}} \iint_{S_{2}} \operatorname{conj}\left(f_{0 t}^{\mathrm{ref}}\right) \dot{u}_{0 t}^{\mathrm{ref}} \mathrm{d} S$

where $f_{0 m}^{\text {inc }}$ means the nodal force of the $m^{\text {th }}$ incident mode, $f_{0 t}^{\text {tra }}$ and $f_{0 t}^{\text {ref }}$ are nodal forces of the $t^{\text {th }}$ transmitted and reflected modes. $\dot{u}$ denotes time derivative, $\delta_{m t}$ is a Kronecker symbol, and the 'conj' signifies complex conjugate. NP represents the number of the scattered guided wave modes and only depends on the incident frequencies for a given thickness of the pipe.

The time-average power of the incident energy flux in $S_{2}$, a source of energy, is also expressed as

$\mathrm{E}_{0 m}^{\mathrm{inc}}=\iint_{S_{2}} \operatorname{conj}\left(f_{0 m}^{\mathrm{inc}}\right) \dot{u}_{0 m}^{\mathrm{inc}} \mathrm{d} S$

Taking into account the energy conservation principle, the incident time-average power must be equal to the sum of energy carried by transmitted and reflected modes, and their ratios should satisfy the condition: 
$\frac{E^{\text {tra }}}{E_{0 m}^{\text {inc }}}+\frac{E^{\text {ref }}}{E_{0 m}^{\text {inc }}}=\widetilde{E}^{\text {tra }}+\widetilde{E}^{\text {ref }}=1$

where $\widetilde{\mathrm{E}}^{\text {tra }}=\frac{\mathrm{E}^{\text {tra }}}{\mathrm{E}_{0 m}^{\text {inc }}}, \widetilde{\mathrm{E}}^{\mathrm{ref}}=\frac{\mathrm{E}^{\mathrm{ref}}}{\mathrm{E}_{0 m}^{\text {inc }}}$.

This equation can be used to check the correctness of scattered fields solved by the developed in-house HFEM in the following section.

\subsection{Validation of in-house HFEM}

In the numerical simulations, the pipeline has been modelled with 20 nodal hexahedron isoparametric elements and its parameters are $d_{\text {ay }}=0.50 \mathrm{~h}$ and $l_{\text {ay }}=0.568 \mathrm{~h}$ shown in Fig. 1. $\mathrm{L}(0,1), \mathrm{L}(0,2)$ and $\mathrm{T}(0,1)$ are chosen as the modes of incident waves. The time-average power of the energy flux can be calculated by in-house HFEM for the different modes of incident waves, respectively. The simulation results are given in Fig. 2. Obviously, the summation of the reflected energy ratio and transmitted energy ratio for the same mode is equal to one, which demonstrates the correctness of the calculation from the energy conservation point of view.

To further verify the correctness of the in-house HFEM, the simulated reflection coefficients for $\mathrm{L}(0,2)$ mode of incident waves have been compared with the ones from Stoyko's work (Stoyko et al., 2014) shown in Fig. 3. It is observed that the results by the developed HFEM are in very good agreement with the data provided by Stoyko.

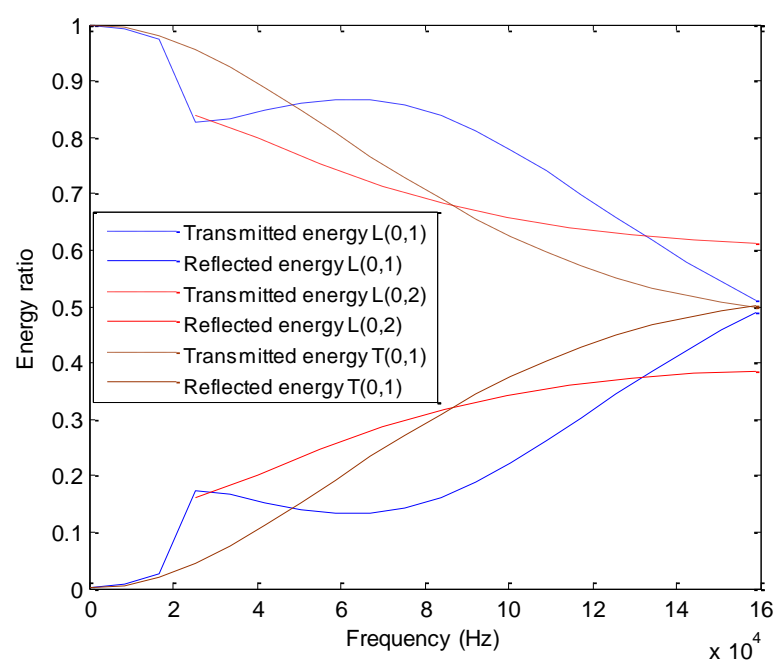

Fig. 2 The time-average power with frequency for different incident modes for a defect profile with

$$
d_{\text {ay }}=0.50 \mathrm{~h} \text { and } l_{\text {ay }}=0.568 \mathrm{~h}
$$




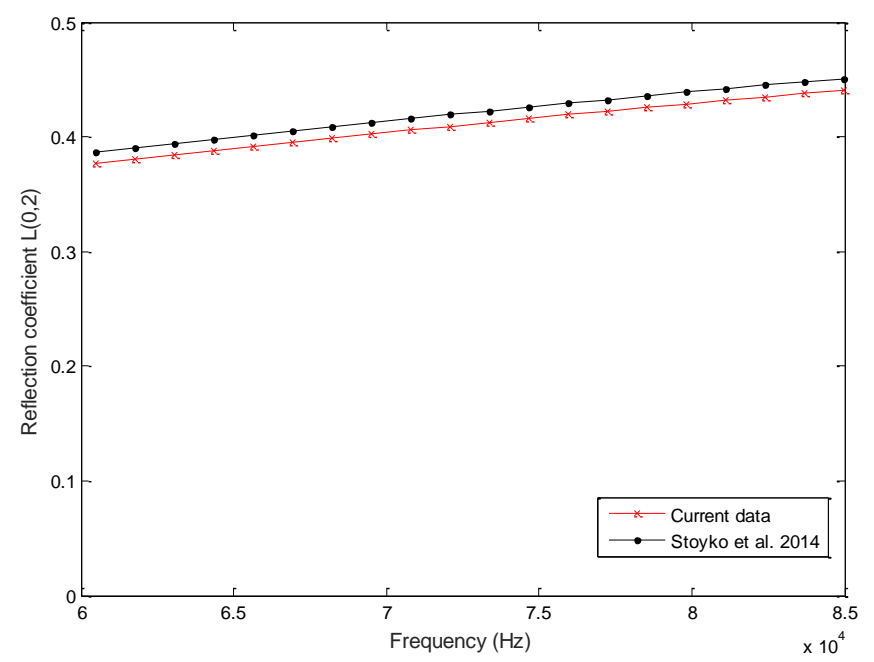

Fig. 3 The absolute values of the reflection coefficients of $\mathrm{L}(0,2)$ for a defect profile with $d_{\text {ay }}=$

$$
0.50 \mathrm{~h} \text { and } l_{\text {ay }}=0.568 \mathrm{~h}
$$

\section{Reconstructing defects by QDFT}

Usually, the scattered wave field is measured using experimental tests or simulated by numerical methods such as finite element method. As introduced in Section 3, an in-house HFEM code is developed in this work to calculate reflection coefficients. To reconstruct defects in plate structures, Wang and Hirose (2012) and Wang et al. (2015) applied a boundary integral equation method with ultrasonic guided waves. In this method, only one point to transmit and receive signals is needed. It is suitable to detect defects resulted from a plate thinning. The main challenge of using boundary integral equation method to reconstruct defects is to derive Green's functions of the corresponding structure. For some simple structures, Green's function can be obtained by mathematical derivations. However, it is impossible to represent the analytical Green's functions of complex structures.

To address this problem, an enhanced method, called as Quantitative Detection of Fourier Transform (QDFT), is developed in this section to efficiently reconstruct defects using guided ultrasonic waves. This approach has the advantage of avoiding the derivation of closed-form Green's functions for general solids and weakens the hypothesis of small existing and emerging defects, as is required to derive Eq. (5) in Section 2. Details of the QDFT method are introduced in the following paragraphs.

Based on Eqs. (7) and (8) for the reconstruction of defects introduced in Section 2, a 
remarkable conclusion can be drawn that the reflection coefficients $C^{\text {ref }}(k)$ and defect function $\eta\left(x_{2}\right)$ can be written as a Fourier transform pair by introducing the term $B(k)$, which only depends on wavenumbers for a given thickness of the structure. Since the reflection coefficients of an unknown defect are usually measured by experimental tests, the major challenge in defect reconstructions is to determine $B(k)$ with sufficient accuracy and efficiency. From a practical point of view, the defect function $H(k)$ in wavenumber domain can be formulated by applying Fourier transform

$H(k)=\int_{-\infty}^{+\infty} \tilde{\eta}\left(x_{2}\right) e^{-i k x_{2}} d x_{2}$

where $\tilde{\eta}\left(x_{2}\right)$ is the defect function in spatial domain, $x_{2}$ is the axis of propagating direction of guided waves, $k$ means the wavenumber of guided waves.

Taking into account Eqs. (7), (8), and (40), the term $\tilde{B}(k)$ of an unknown defect can be obtained as follows:

$\tilde{B}(k) \approx \frac{H(k)}{C^{\operatorname{ref}}(k)}$

According to the foregoing derivations, an enhanced method to reconstruct unknown defects using the term $B_{0}(k)$ of a known defect, with which the term $\tilde{B}(k)$ of an unknown defect will be replaced, is proposed and it is called as Quantitative Detection of Fourier Transform (QDFT). The detailed procedure of QDFT is described below:

Step 1: To apply Fourier transform on the known defect $\eta_{0}\left(x_{2}\right)$ and then, obtain its Fourier transform pair $H_{0}(k)$ in wavenumber domain;

Step 2: To calculate $B_{0}(k)$ in use of Eq. (41) and it should be noted that reflection coefficients $C_{0}^{\text {ref }}(k)$ of the known defect is computed by in-house HFEM;

Step 3: To obtain the reflection coefficients $C^{\text {ref }}(k)$ of the unknown defect by experimental tests;

Step 4: To reconstruct the unknown defects using the results $\left(B_{0}(k)\right.$ and $\left.C^{\text {ref }}(k)\right)$ obtained from Step 2 and Step 3.

$\eta_{1}\left(x_{2}\right) \approx \frac{1}{2 \pi} \int_{-\infty}^{+\infty} C^{\mathrm{ref}}(k) B_{0}(k) e^{i k x_{2}} d k$

It is noted that the closer the profile of the known defect matches the shape of the unknown flaw, the better the reconstruction of defects is. Practically, the results of representing defects 
are of satisfactory quality when the unknown $\tilde{B}(k)$ is replaced by $B_{0}(k)$ of a known rectangular defect for initial calculation in a process of defect reconstruction.

\section{Processing the signal of reference model and numerical experiments}

\subsection{Validation of the proposed QDFT method}

In this section, three different sizes of single rectangular axisymmetrical defects in pipelines are analyzed using in-house HFEM to determine reflection coefficients, which are then used for the following reconstruction of defects. The defect parameters are shown in Fig. 4: Case $1, l_{\mathrm{ay}}=0.7778 \mathrm{~h}$ and $d_{\mathrm{ay}}=0.3333 \mathrm{~h} ;$ Case $2, l_{\mathrm{ay}}=1.40 \mathrm{~h}$ and $d_{\mathrm{ay}}=0.1667 \mathrm{~h}$; Case $3, l_{a y}=1.40 \mathrm{~h}$ and $d_{a y}=0.3333 \mathrm{~h}$. The wall thickness in all three cases is $\mathrm{h}=$ $0.0056 \mathrm{~m}$. The corresponding reflection coefficients in each case are computed using in-house HFEM described in Section 3 and presented in Fig. 5.

(a)

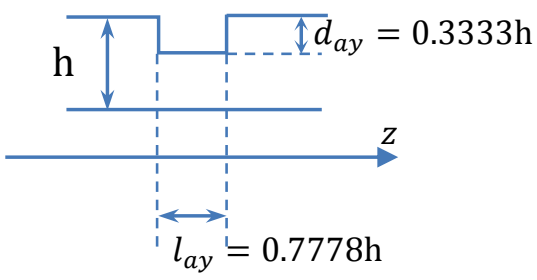

(b)

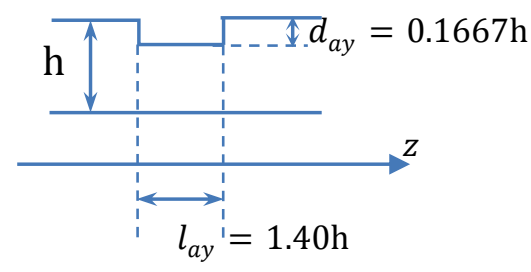

(c)

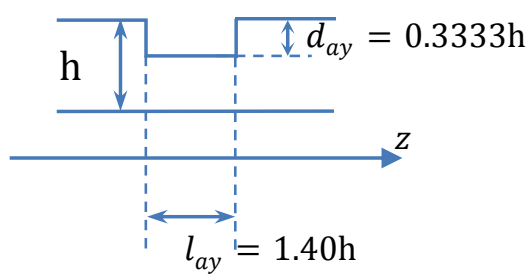

Fig. 4 Three different sizes of single rectangular defects: (a) Case 1, (b) Case 2, and (c) Case 3 


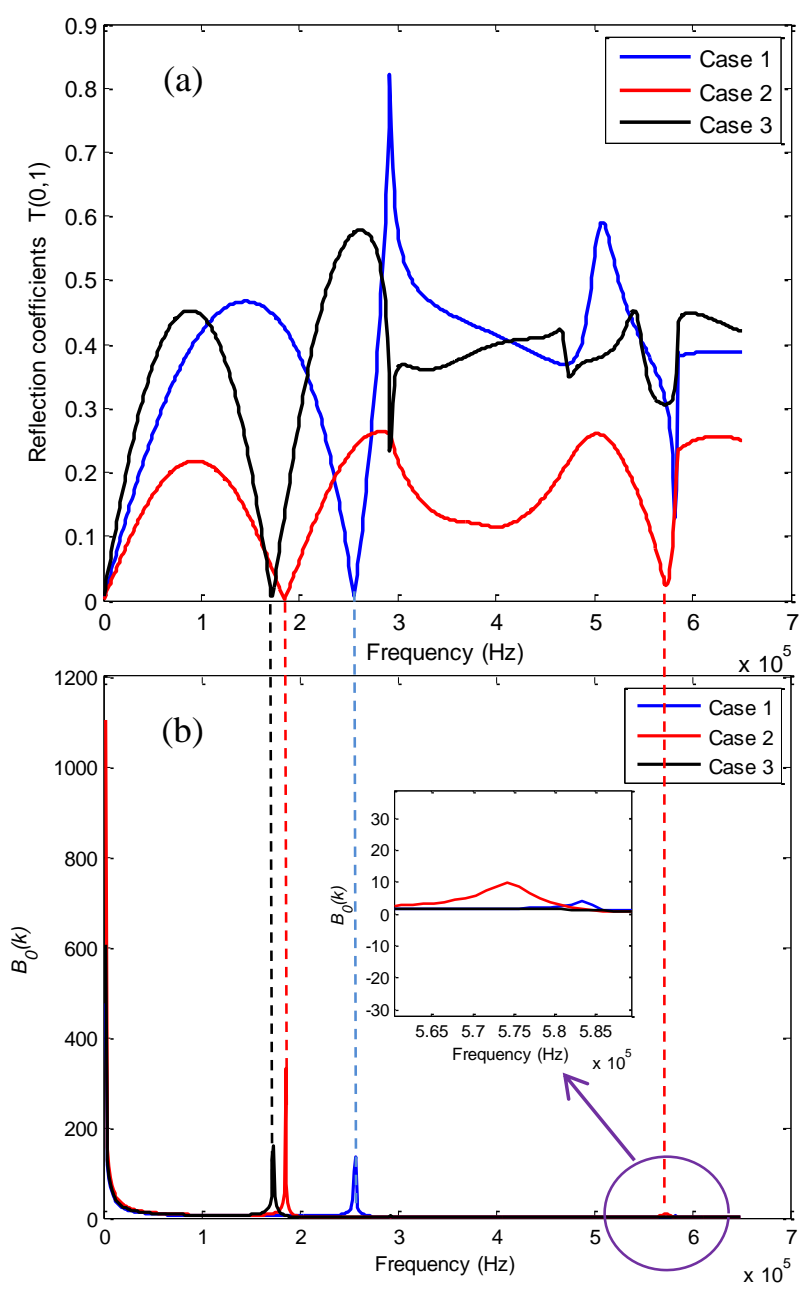

Fig. 5 Absolute values of reflection coefficients $C_{0}^{\text {ref }}(a)$, and $B_{0}$ (b) as a function of frequency

It is observed that the shorter the defect length $l_{\text {ay }}$ is, the smaller the defect height $d_{a y}$ is, and the wider the first peak bandwidth gets. However, the length of a defect $l_{\text {ay }}$ is a major factor which leads to a high initial peak bandwidth. The reflection coefficients $C_{0}^{\text {ref }}(k)$ approach to zeros in Fig. 5(a), which comply with the positions of cuspidal points on the curve $B_{0}(\mathrm{k})$ shown in Fig. 5(b). Actually, the contributions of $B_{0}(\mathrm{k})$ to the reconstruction of defects at the cuspidal points are very little due to the zero values of the corresponding reflection coefficients and this can be proved using Eq. (8). It is concluded that the reconstruction accuracy is influenced by the interaction of $B_{0}(k)$ and $C_{0}^{\text {ref }}(k)$, each of which has been affected by the shape of a defect in terms of magnitudes.

In the proposed QDFT approach, the information of the real flaw is given as: $l_{\text {ay }}=$ $1.40 \mathrm{~h}$ and $d_{\mathrm{ay}}=0.3333 \mathrm{~h}$. The curve $B_{0}^{(1)}(k)$ of Case 2 described in Section 4 is initially considered as the reference curve $B_{0}(k)$ to reconstruct the unknown defect. The 
reconstructed result is shown in Fig. 6. Employing Fourier transform, the fluctuation of the predicted defect within the region of no defect along the pipe is converted in frequency domain shown in Fig. 7. It is noted that the fluctuation in the non-defective region actually results from the contribution of the cuspidal points on the red curve $B_{0}^{(1)}(k)$ to the construction of defects because the frequencies (about $173 \mathrm{KHz}$ and $574 \mathrm{KHz}$ ) of such noise in Fig. 7 are exactly the same as the ones in Fig. 5. In order to obtain a clear profile of the defect in the reconstruction process, the zero values of $B_{0}(k)$ are assigned at the frequencies near the regions of the cuspidal points, where one fifth of the maximum $\mathrm{B}_{0}(\mathrm{k})$ at each cuspidal point is observed. Fig. 8 shows a more precise defect depth as compared with the defect profile in Fig. 6. Although the defect depth predicted is slightly deeper than the real defect depth, a good agreement between the two results can be seen with acceptable accuracy. The defect location and shape can be preliminarily determined by QDFT approach for reconstruction. As described in Section 4, the main reason for the discrepancy between two curves in Fig. 8 is that the reference defect used in Case $2\left(l_{a y}=1.40 \mathrm{~h}\right.$ and $\left.d_{a y}=0.1667 \mathrm{~h}\right)$ has a remarkably different depth compared with the real defect $\left(l_{a y}=1.40 \mathrm{~h}\right.$ and $d_{a y}=$ $0.3333 h)$.

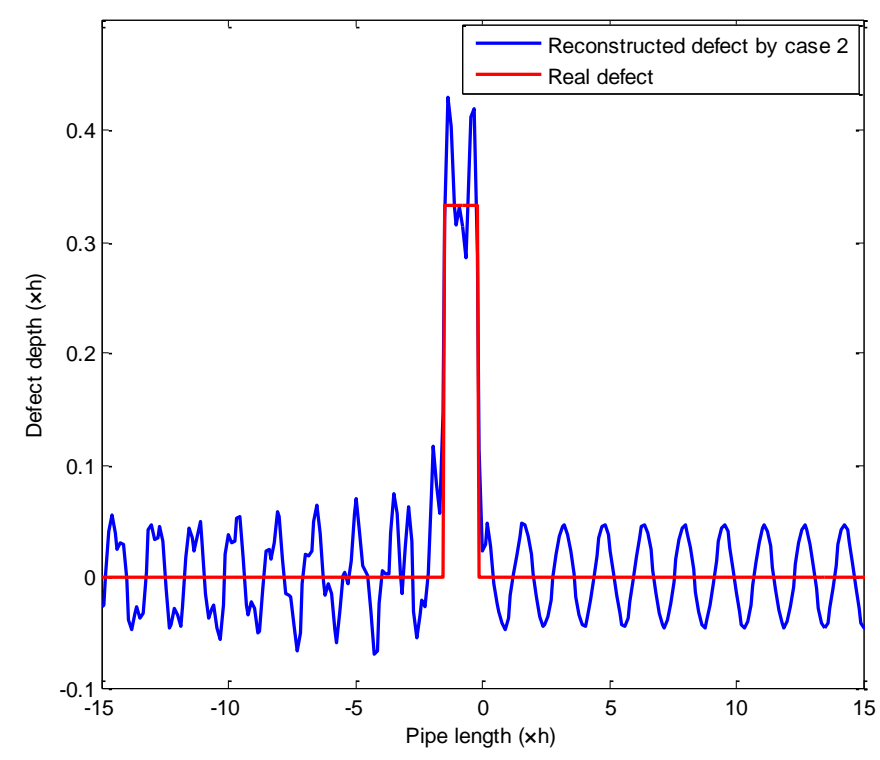

Fig. 6 Reconstructing the defect using Case 2 as a reference model 


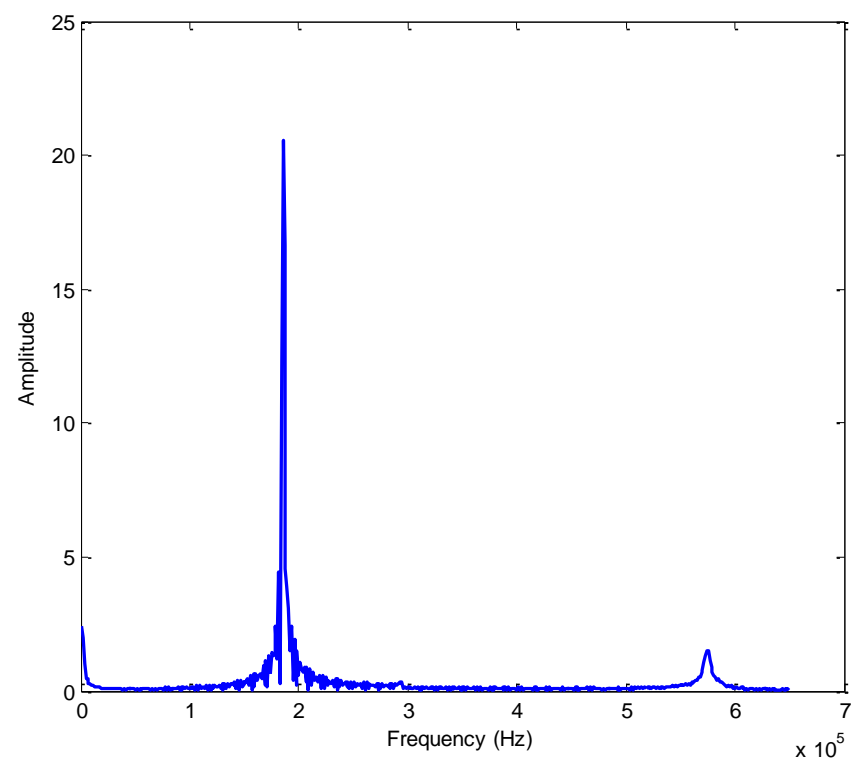

Fig. 7 Fourier transform of defect depth in the non-defective region in Fig.6

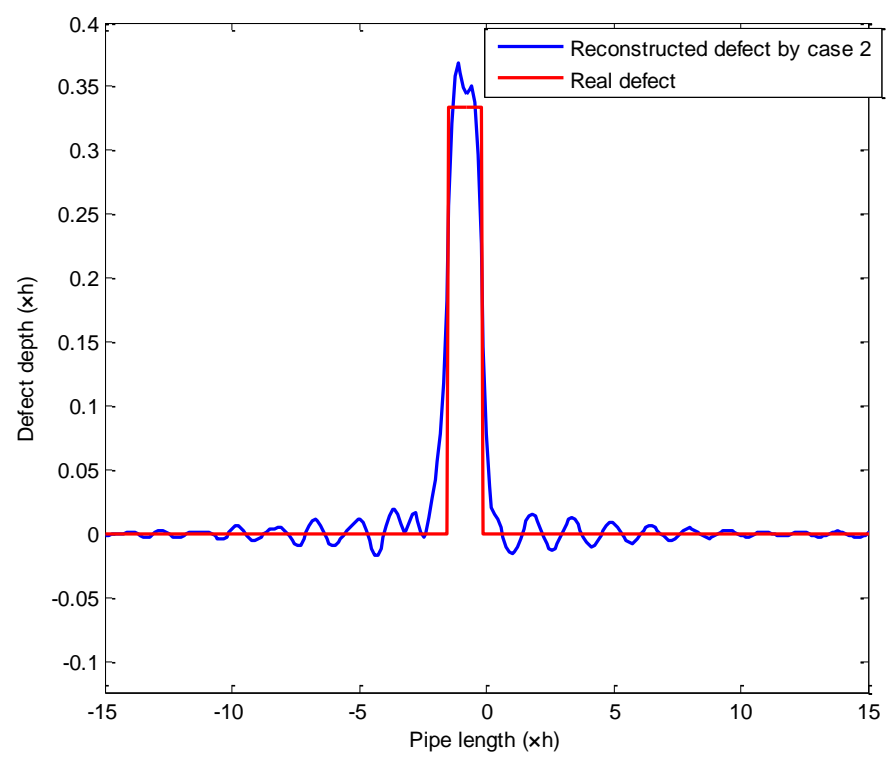

Fig. 8 Reconstructing defect by filtered $B_{0}(k)$ of Case 2

\subsection{Effect of $B_{0}(k)$ on the accuracy of reconstruction of defects}

In Fig. 9, four common types of defects, i.e., a single rectangular flaw, a double-stepped flaw, a penta-stepped flaw, and a double rectangular flaw, have been investigated. To effectively reflect the incident guided waves from defects in pipe structures, the fundamental torsional mode $\mathrm{T}(0,1)$, which is a non-dispersive mode and its phase velocity is equal to the transverse velocity, is generated and detected by ultrasonic experiments. 
(a)

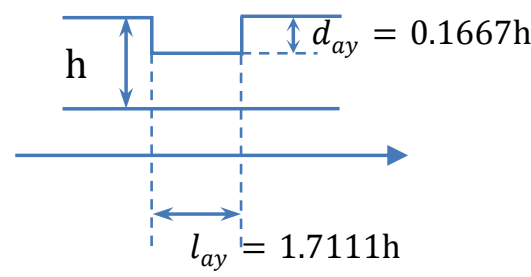

(c)

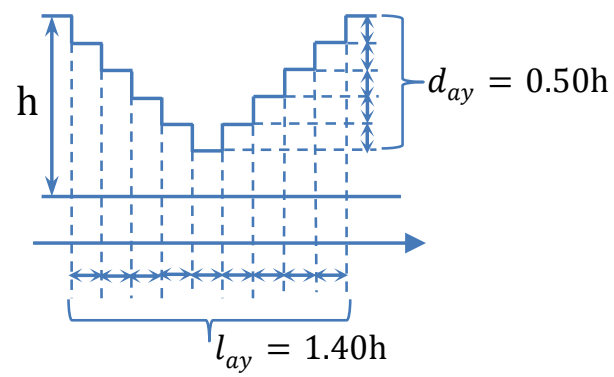

(b)

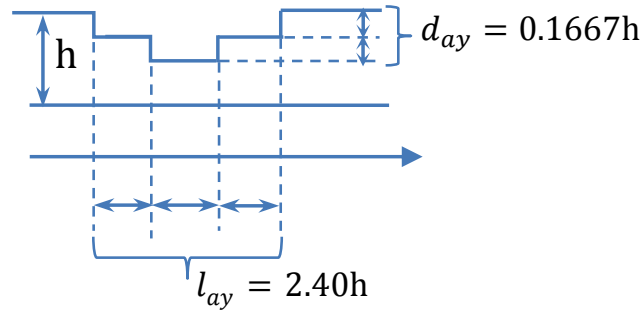

(d)

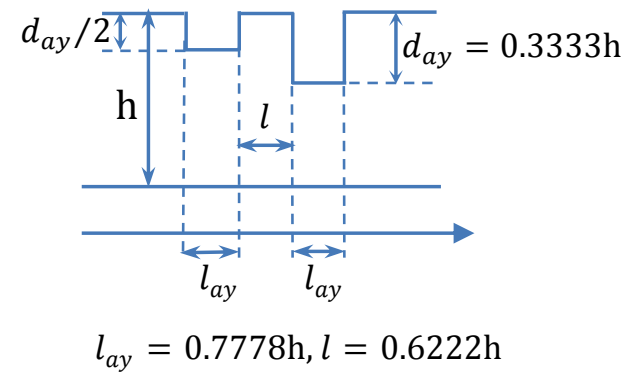

Fig. 9 Defect models to be reconstructed: (a) a single rectangular defect, (b) a double-stepped defect, (c) a penta-stepped defect, and (d) double rectangular defects

To further assess high-efficiency and accuracy of the developed fast QDFT approach for detecting surface defects in pipeline structures, each of three different defects shown in Fig. 4 is used as the reference model to reconstruct four types of defects, whose shapes are given in Fig. 9: (a) a single rectangle defect with $l_{\mathrm{ay}}=1.7111 \mathrm{~h}$ and $d_{\mathrm{ay}}=0.1667 \mathrm{~h}$, (b) a double-stepped defect with the step depth of $0.0833 \mathrm{~h}$ and $l_{\text {ay }}=2.40 \mathrm{~h}$, (c) a five-stepped defect with the step depth of $0.10 \mathrm{~h}$ and $l_{\text {ay }}=1.40 \mathrm{~h}$, and (d) double rectangle defects, whose widths and depths are $l_{\text {ay }}=0.7778 \mathrm{~h}, d_{\text {ay }}=0.1667 \mathrm{~h}$ and $l_{\text {ay }}=0.6222 \mathrm{~h}, d_{\text {ay }}=$ $0.3333 \mathrm{~h}$, respectively.

Fig. 10 shows results obtained from each of three reference models have been compared with the real defect. Since the defect shape of the reference model in Case $1\left(l_{a y}=\right.$ $0.7778 \mathrm{~h}$ and $d_{a y}=0.3333 \mathrm{~h}$ ) is the most different from the given single rectangle defect ( $l_{\text {ay }}=1.7111 \mathrm{~h}$ and $d_{\mathrm{ay}}=0.1667 \mathrm{~h}$ ), it is obviously observed that the accuracy of reconstruction in Case 1 is the worst among three cases. Either of reference models in Case 2 or Case 3 has more capability to provide $B_{0}(k)$ with high accuracy for reconstruction of the unknown defect using QDFT. In general, all three reference models can be used to reconstruct the defect with acceptable precision.

For a double-stepped defect shown in Fig. 9(b), the most accurate information of $B_{0}(k)$ obtained from the reference model in Case 2 for reconstruction can be observed in Fig. 11 
with respect to the shape and depth of the unknown defect. Similarly, reconstruction by the reference model in Case 1 can be assessed with the best accuracy among all three reference models due to its closest geometric parameters to the representative feature of the five-stepped flaw in Fig. 12. This can also help understand why the construction by Case 3 has better accuracy than the one by Case 2 .

The most challenging problem is to reconstruct the double rectangle defects shown in Fig. 9(d). As the aforementioned discussion, the representative feature of double rectangle defects is described as $l_{\text {ay }}=0.7778 \mathrm{~h}$ and $d_{\text {ay }}=0.3333 \mathrm{~h}$, which can be reflected by the reference model in Case 1. This is the reason why the best result relating to the reference model in Case 1 is observed in Fig. 13. The good agreement of predicted results with the real double rectangle defects proves the correctness and efficiency of the proposed QDFT approach for nondestructive detection and reconstruction of the defects in the complex structures.

Throughout these simulations analyzed, it is concluded that three indexes including the maximum depth of the defect, the defect area, and the equivalent width of the defect can be proposed to judge the similarity between the reference model and unknown defect. It is also noted that the equivalent width of the defect should be calculated using the maximum depth of the defect divided by the defect area.

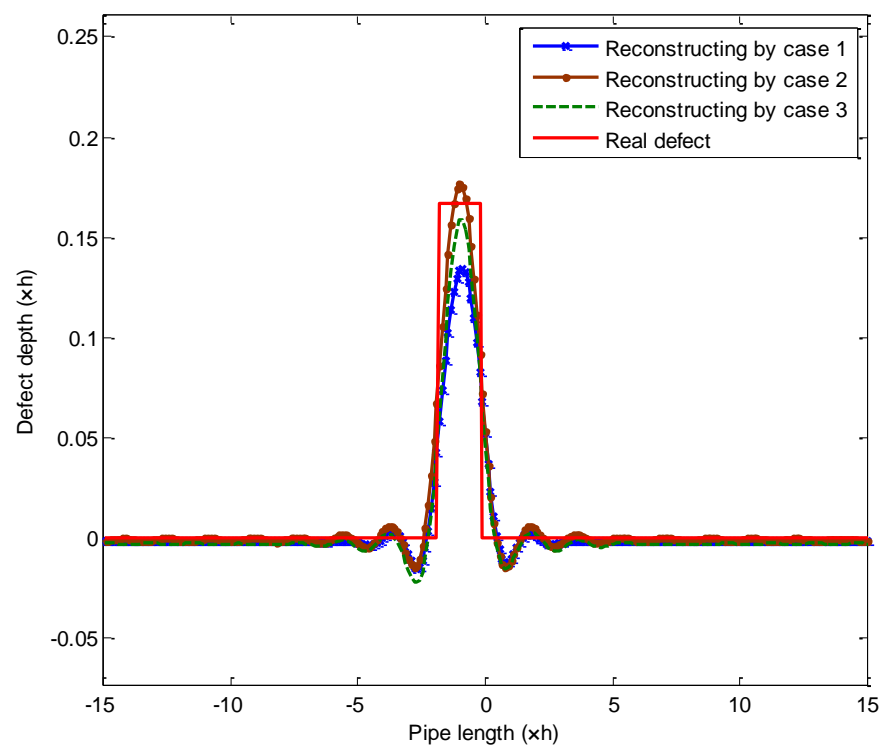

Fig. 10 Reconstructing the single rectangular defect by $B_{0}(k)$ in Cases 1, 2 and 3, respectively 


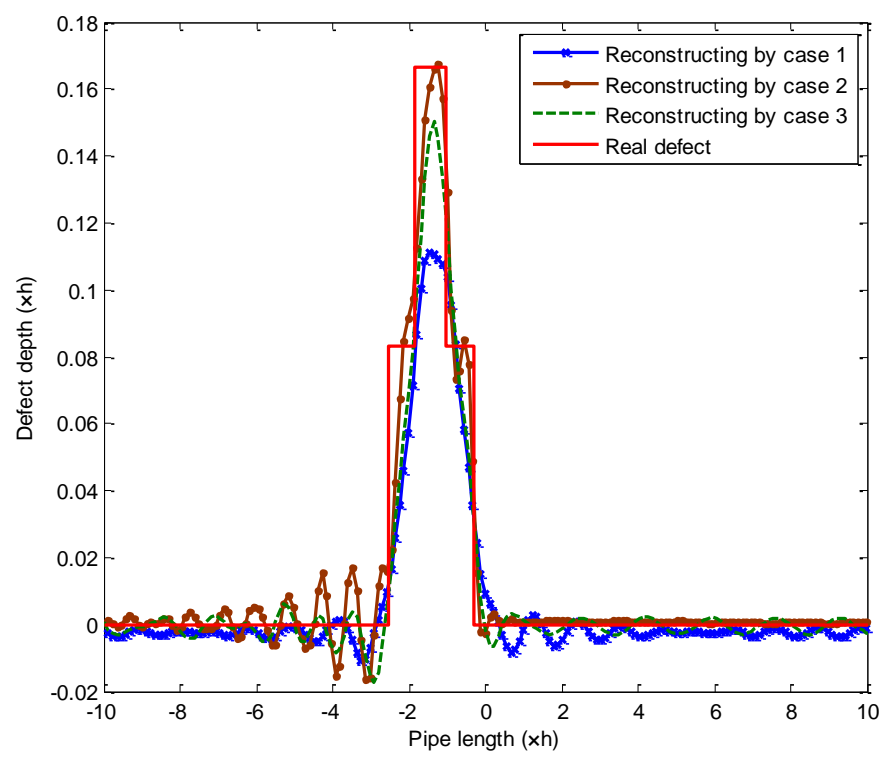

Fig. 11 Reconstructing the double-stepped defect based on the reference models of Case 1,2 and 3, respectively

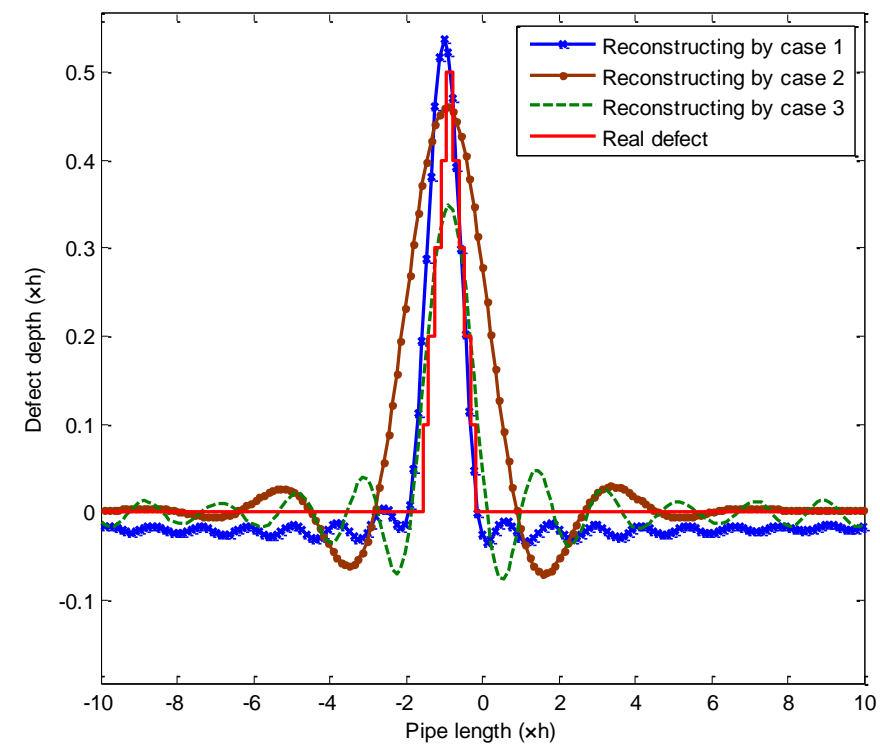

Fig. 12 Reconstructing the penta-stepped defect based on the reference models of Case 1, 2 and 3, respectively 


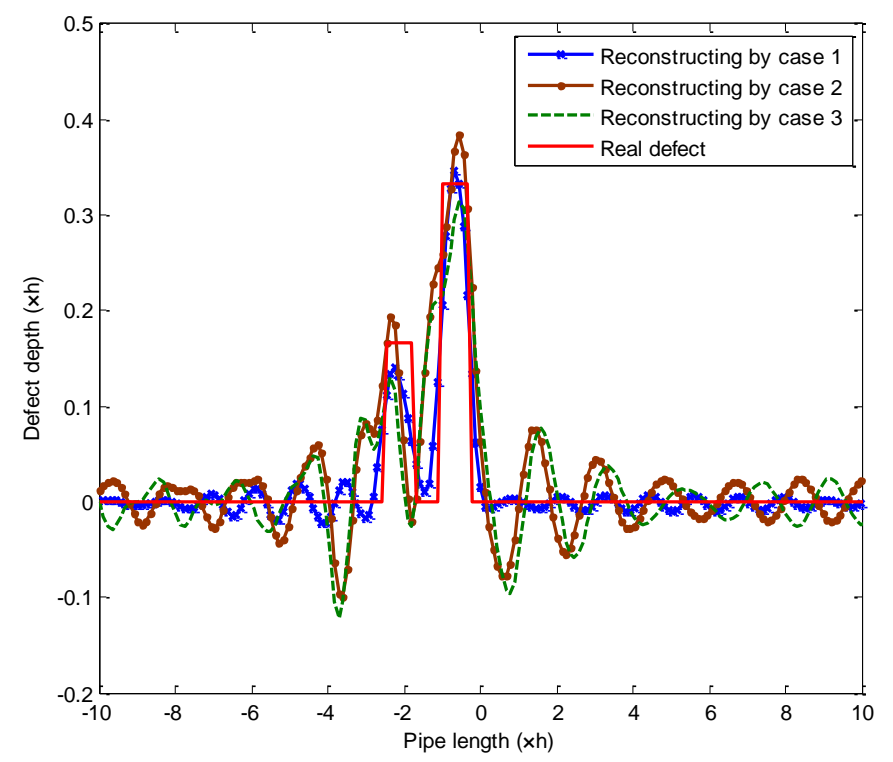

Fig. 13 Reconstructing the double rectangular defects based on the reference models of Case 1, 2 and 3, respectively

\section{Conclusions}

Quantitative Detection of Fourier Transform (QDFT), a novel approach capable of providing high level of accuracy and efficiency for nondestructive detection of defects, is proposed in this paper. Based on boundary integral equations and Fourier transform, this method helps establish an explicit connection between reflection coefficients of guided waves and the defect shape by introducing a wavenumber-dependent function $B(k)$, which is applied to reconstruct the unknown defects in use of the reference defect model. It is certain that signal processing is indispensable to improve the precision of this procedure. To demonstrate the correctness and efficiency of this developed approach, three types of defects: a single rectangular flaw, double rectangular flaws and stepped deep flaws, are examined by a comparative study on the reconstruction precision of defects between different reference models and real defects. Further analyses have shown that the maximum depth of the defect, the defect area, and the equivalent width of the defect are three indexes to judge the similarity between the reference model and unknown defect, which also control the reconstruction accuracy. It is noted that QDFT is suitable for solving general two-dimensional surface defect problems and also avoids the necessity of deriving closed-form Green's functions for complex defected structures. The proposed approach contributes to the characterization of surface 
defects and would also broaden the scope of nondestructive defect inspection using ultrasonic guided waves.

Future investigations are required on enhancement of the QDFT approach by iterative analyses in order to adopt a general reference model for high accurate reconstruction of two-dimensional surface defects in complex structures.

\section{Acknowledgements}

This research was supported by the National Natural Science Foundation of China (nos. 11502108, 11232007, 51405225, and 11611530686), the Program for New Century Excellent Talents in Universities (no. NCET-12-0625), the Natural Science Foundation of Jiangsu Province (nos. BK20140037 and BK20140808), the Fundamental Research Funds for Central Universities (no. NE2013101), and the project funded by the Priority Academic Program Development of Jiangsu Higher Education Institutions (PAPD). The authors would like to thank many colleagues at Nanjing University of Aeronautics and Astronautics for their valuable suggestions during the preparation of this paper, and the reviewers for their instructive comments on the paper.

\section{References}

Argatov, I., Butcher, E.A., 2011. On the separation of internal and boundary damage in slender bars using longitudinal vibration frequencies and equivalent linearization of damaged bolted joint response. Journal of Sound and Vibration 330(13), 3245-3256.

Batenburg, K., Sijbers, J., 2011. DART: A practical reconstruction algorithm for discrete tomography. IEEE Trans Image Process 20 (9), 2542.

Blackledge, M., Burge, R.E., Hopcraft, K.I., Wombel, R.J., 1987. Quantitative diffraction tomography. I. Pulsed acoustic fields. Journal of Physics. D: Applied Physics 20 (1), 1-10.

Blackledge, M., Burge, R.E., Hopcraft, K.I., Wombel, R.J., 1987. Quantitative diffraction tomography. II. Pulsed elastic waves. Journal of Physics. D: Applied Physics 20 (1), 11.

Chang, Q., Peng, T., Liu, Y., 2018. Tomographic damage imaging based on inverse acoustic wave propagation using k-space method with adjoint method. Mechanical Systems and Signal Processing 109, 379-398. 
Cook, R., 1981. Concepts and applications of finite element analysis. second ed. John Wiley and Sons, New York, N.Y., USA.

Devaney, A.J., 1984. Geophysical Diffraction Tomography. IEEE Transactions on Geoscience and Remote Sensing GE-22 (1), 3-13.

Ditri, J.J., Rose, J.L., 1992. Excitation of guided elastic wave modes in hollow cylinders by applied surface tractions. J. Appl. Phys 72 (2), 2589-2597.

Dobson, D.C., Santosa, F., 1998. Nondestructive evaluation of plates using eddy current methods. International Journal of Engineering Science 36(4), 395-409.

Duan, W., Kirby, R., 2015. A numerical model for the scattering of elastic waves from a non-axisymmetric defect in a pipe. Finite Elem. Anal. Des. 100(C), 28-40.

Hay, T.R., Rose, J. L., 2002. Flexible PVDF comb transducers for excitation of axisymmetric guided waves in pipe. Sensors and Actuators. A: Physical 100 (1), 18-23.

Hosoya, N., Yoshinaga, A., Kanda, A., Kajiwara, I., 2018. Non-contact and non-destructive Lamb wave generation using laser-induced plasma shock wave. International Journal of Mechanical Sciences 140, 486-492.

Huthwaite, P., Simonetti, F., 2013. High-resolution guided wave tomography. Wave Motion 50 (5), 979-993.

Huthwaite P., 2014. Evaluation of inversion approaches for guided wave thickness mapping. Proceedings of the Royal Society. A: Mathematical, Physical and Engineering Sciences 470 (2166).

Jing, R., Ratassepp, M., Zheng, F., 2017. Investigation of the reconstruction accuracy of guided wave tomography using full waveform inversion. Journal of Sound \& Vibration 400, $317-328$

Laroque, S.J., Sidky, E.Y., Pan, X., 2008. Accurate image reconstruction from few-view and limited-angle data in diffraction tomography. Journal of the Optical Society of America 25 (7), 1772-1782.

Leonard, K.R., Malyarenko, E.V., Hinders, M.K., 2002. Ultrasonic Lamb wave tomography. Inverse Problems 18 (6), 1795-1808.

Liu, Z., He, C., Wu, B., Wang, X., Yang, S., 2006. Circumferential and longitudinal defect detection using $\mathrm{T}(0,1)$ mode excited by thickness shear mode piezoelectric elements. 
Ultrasonics 44 (1), e1135-e1138.

Marzani, A., 2008. Time-transient response for ultrasonic guided waves propagating in damped cylinders. International Journal of Solids and Structures 45(25-26), 6347-6368.

Minnaar, K., Zhou, M., 2004. A novel technique for time-resolved detection and tracking of interfacial and matrix fracture in layered materials. J. Mech. Phys. Solids 52(12), 2771-2799.

Mountassir, M.E., Yaacoubi, S., Mourot, G., Maquin, D., 2018. Sparse estimation based monitoring method for damage detection and localization: A case of study. Mechanical Systems and Signal Processing 112, 61-76.

Mu, J., Zhang, L., Rose, J.L., 2007. Defect circumferential sizing by using long range ultrasonic guided wave focusing techniques in pipe. Nondestructive Testing \& Evaluation 22(4), 239-253.

Psychas, I.D., Schauer, M., Böhrnsen, J.U., Marinaki, M., Marinakis, Y., Langer, S., Stavroulakis, G., 2016. Detection of defective pile geometries using a coupled FEM/SBFEM approach and an ant colony classification algorithm. Acta Mechanica 227(5), $1279-1291$.

Rattanawangcharoen, N., Zhuang, W., Shah, A.H., Datta, S.K., 1997. Axisymmetric Guided Waves in Jointed Laminated Cylinders. Journal of Engineering Mechanics 123(10), $1020-1026$.

Schmerr, L.W., 1998. Fundamentals of Ultrasonic Nondestructive Evaluation. Plenum Publishing Corporation.

Sheppard, C.J.R., Shan, S.K., 2010. 3D Imaging with Holographic Tomography. AIP Conference Proceedings 1236 (1), 65-69.

Siqueira, M.H., Gatts, C.E., da Silva, R.R., Rebello, J.M., 2004. The use of ultrasonic guided waves and wavelets analysis in pipe inspection. Ultrasonics 41 (10), 785-797.

Stoyko, D. K., Popplewell, N., Shah, A. H., 2014. Detecting and describing a notch in a pipe using singularities. International Journal of Solids and Structures 51(15-16), 2729-2743.

Wang, B., Qian, Z., Hirose, S., 2015. Inverse Shape Reconstruction of Inner Cavities Using Guided SH-Waves in a Plate. Shock and Vibration.

Wang, B., Hirose, S., 2012. Shape Reconstruction of Plate Thinning Using Reflection 
Coefficients of Ultrasonic Lamb Waves: a Numerical Approach. ISIJ International 52(7), $1320-1327$.

Wang, X.Q., Wong, W.O., Cheng, I., 2008. Modal power flow with application to damage detection. International Journal of Engineering Science, 47(2009), 512-523.

Zhuang, W., Shah, A.H., 1999. Elastodynamic Green's Function for Laminated Anisotropic Circular Cylinders. Journal of Applied Mechanics 66(3), 665-674. 